\title{
Transmission Policies for Data Aggregation using Cooperate Node in Wireless Sensor Networks
}

\author{
Shrikant A. Shinde ${ }^{1}$, Shailaja N. Uke ${ }^{2}$ \\ ${ }^{1}$ Department of Information Technology, Smt. Kashibai Navale College of Engineering, Pune, India \\ ${ }^{2}$ Professor, Department of Information Technology, Smt. Kashibai Navale College of Engineering, Pune, India
}

\begin{abstract}
Wireless Sensor Networks (WSNs) consist of sensor nodes and the sensor nodes are capable of collecting, sensing and gathering data from the environment. These networks have extensive application in disaster management, habitat monitoring, security, and military, etc. Wireless sensor nodes are very small in size and very low battery power and have limited processing capability. Data aggregation is a very important technique in WSNs and helps in reducing the energy consumption by eliminating redundancy. The main aim of data aggregation is to collect and gather data in an energy efficient manner and due to this the network lifetime is improved. One data aggregation method in a WSN is sending local representative data to the Sink node based on the spatial-correlation of sampled data. Based on this correlation degree, a data density correlation degree (DDCD) clustering method is presented in detail so that the representative data have a low distortion on their correlated data in a WSN. The proposed system uses a co-operate node or data centre node which cooperates among the sensor nodes in a particular area of WSN and which improves transmission policies as well as energy efficiency. To design a modified data density correlation degree clustering algorithm for energy balanced network by using clustered data aggregation methodology. The simulation results show that the resulting representative data achieved using the proposed modified data density correlation degree clustering method have better throughput, packet delivery ratio, dropping ratio, delay, normalized overhead and average energy consumption than those achieved using the data density correlation degree clustering method.
\end{abstract}

Keywords: Data aggregation, data density, correlation degree, energy efficiency, wireless sensor networks.

\section{Introduction}

Wireless Sensor Networks (WSNs) are the collection of number of small nodes for performing some functions as processing, gathering sensory information and to communicate with other connected nodes in the network. Features of WSN are homogeneous devices, no wired infrastructure, potential multi hop routes, dispersed large network size and self-organization. The type nodes may be mobile or stationary and all nodes act as routers. Intermediate nodes are responsible to establish communication between two unconnected nodes [2]. Every node in network that falls inside the communication range of a node is considered to be reachable from every node. WSN have many applications like Industrial control and monitoring, Home automation, Security, Military sensing and directing, Consumer electronics, Asset tracking and supply chain management, Intelligent Agriculture, commercial applications, Fire alarming, Environmental monitoring and health monitoring etc.

Data aggregation is a very important technique in WSNs and helps in reducing the energy consumption by eliminating redundancy. Data aggregation is the process of gathering and aggregating the data. Data aggregation is one of the fundamental processing procedures for saving the energy and is an effective way to save the limited resources as well energy. The main purpose of data aggregation is to gather, collect and aggregate the data in an energy effective manner so that the network lifetime is enhanced [2]. Data aggregation improves the lifetime of sensor nodes by eliminating redundant data transmission. The data transmission uses a multi-hop fashion in which each node transfers data to the neighbor node nearer to the sink.
The rest of the paper is organized as follows: Section II summarizes the related work. Section III presents the proposed methodology. Section IV summarizes experimental setup and required parameters for simulation. In Section $\mathrm{V}$ results and discussion are described and Section VI states the conclusion and future scope.

\section{Related Work}

In current work on modeling spatial correlation, spatial correlation models are mainly based on the locations of sensor nodes or statistical features of sensor nodes' data. In [1], a data density correlation degree (DDCD) clustering method based on correlation degree is given in this paper so that the representative data have a less distortion on their correlated data in a WSN. The advantage is that the distance between representative data and the data center of its correlated data which are in its $\varepsilon$-neighborhood has significant influence on the value of DDCD. The Cooperative Multiple-Input-Multiple-Output (CMIMO) is a method which is adopted in cluster-based WSN to bring in cooperation among the sensor nodes in a particular cluster. Data Aggregation is a method which is combined with CMIMO to minimize the energy cost further by decreasing the amount of data in transit [2]. A.K.M. Azad and Joarder Kamruzzaman [3], proposes a transmission scheme and determines the optimal ring thickness and hop size by preparing network lifetime as an optimization problem. Two other variations also presented by redefining the optimization problem considering: associated hop size variation by sensors over lifetime along with optimal duty cycles, and definite set of hop sizes for sensors in each ring. In [4], the DOC protocol is given and they have proved that there exists optimization algorithm that can discover an optimal rate allocation within each cluster to reduce the intra-cluster communication cost and have presented an 


\section{International Journal of Science and Research (IJSR) \\ ISSN (Online): 2319-7064}

Index Copernicus Value (2015): 78.96 | Impact Factor (2015): 6.391

intra-cluster coding protocol to perform locally SlepianWolf coding within a single cluster.

In [5], $\alpha$-local spatial clustering algorithm for sensor networks is proposed. By measuring the spatial correlation between data sampled by different sensors, the algorithm constructs a dominating set as the sensor network backbone used to realize the data aggregation based on the information description/summarization performance of the dominators. In EECC scheme, a cooperative reply is performed at each hop by the best-suited node selected from those that have successfully overheard the transmitted packet. This new scheme takes advantage of cooperative transmission to enhance the routing robustness against link unreliability [6]. In [7], a distributed clustering algorithm is given; Energyefficient Clustering that calculates suitable cluster sizes depending on the hop distance to the data sink while achieving equalization of node lifetimes and minimized energy consumption levels.

Data coming from various sensor nodes aggregated as if they are about the same attribute of the situation when they reach the same routing node on the way back to the sink. Data aggregation is a widely used method in wireless sensor networks. The security issues, data confidentiality, and integrity, in data aggregation become important when the sensor network is used in a hostile environment. Data aggregation is a method of gathering the sensor data using aggregation approaches [8]. One of the important security challenges for data aggregation is that the aggregators present clear data at the aggregation level. Therefore, this aggregation level is exposed to attacks by intruders. Existing research has addressed this problem and presented solutions by considering static node topology of WSN. The advantage of this approach is that it tackles the security problems that arise in dynamic node WSN [9]. In [10], to adjust the performance between aggregation time and network lifetime, a scheme called time-window and Voronoipartition based aggregation scheduling (TVAS) is proposed in this paper.

In [11], the proposed technique selects cooperative nodes in a network using a multi-criterion based algorithm, and data is transmitted through multi-hop. Through the proposed technique unnecessary transmissions by the non-cooperative nodes are avoided. The disadvantage of this technique is that the distance criteria have not been considered.

In [12], DDCD clustering method is developing a method which could confirm the parameters modified to the real sampled data, particularly the data threshold has major effect on clustering performance. Furthermore, in data transmitting process, the energy of sensor nodes should be considered to construct an energy balanced networks.

In [13], DDCD (Data Density Correlation Degree) algorithm on wireless sensor network is works as a middleware for aggregating data sustained by a more number of nodes within a network. The problem encountered in the recent past was of the more battery power consumption. Therefore, this paper proposed the efficient and effective mechanism of energy efficient procedures for data aggregation in wireless sensor network and increase the network lifetime.
In [14], author gives new scheme related to clustering for data aggregation called "Efficient cluster head selection scheme for data aggregation in wireless sensor network" (ECHSSDA), also we compare our propose scheme to the LEACH clustering algorithm. Comparison is based on the energy consumption, cluster head selection and cluster formation.

In [15], author study the problem of maximizing network lifetime through balancing energy consumption for uniformly deployed data-gathering sensor network and formulate the energy consumption balancing problem as an optimal transmitting data distribution problem by combining the ideas of corona-based network division and mixedrouting strategy together with data aggregation. The approach for computing the optimal number of coronas in terms of maximizing network lifetime is also presented. Based on the mathematical model, an energy-balanced data gathering (EBDG) protocol is designed and the solution for extending EBDG to large-scale data-gathering sensor networks is also presented.

\section{Proposed Methodology}

\section{A. Overview}

The WSN is modeled by undirected graph $\mathrm{G}=(\mathrm{V}, \mathrm{E})$, Where $\mathrm{V}$ is the set of all sensor nodes in the WSN; $\mathrm{E}$ is the edge set consisting of all links in the WSN. The antenna of sensor node $i(i \in \mathrm{V})$ is an Omni-directional antenna, with a communication radius of $\alpha(i)$. Let $\mathrm{N}(i)$ be the set of sensor nodes within the circle of the communication radius of $i$. In cluster-based data aggregation networks, every cluster head sends aggregated data obtained from its member nodes to the sink node by one hop or multi-hops. Fig. 1 shows the architecture of proposed system.

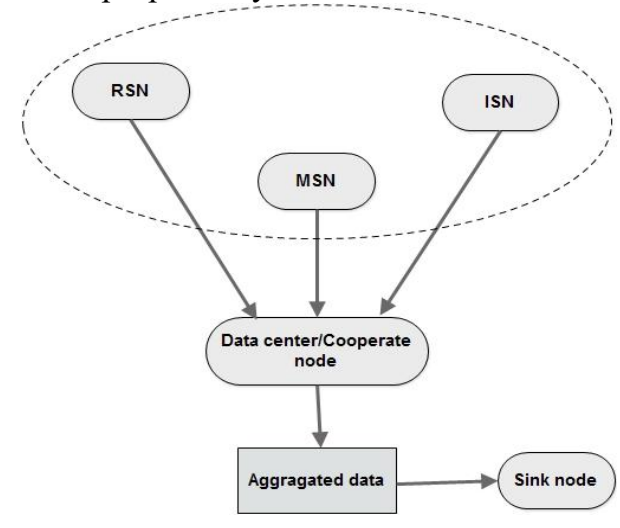

Figure 1: Proposed system architecture

As shown in fig.1, sensor nodes are classified into three types: representative sensor nodes (RSN), isolated sensor nodes (ISN) and member sensor nodes (MSN). The RSN and ISN are responsible for sensing and sending sampled data to the sink node. While the MSN just transmit sampled data collected by RSN or ISN, or do nothing. The data center/cooperate node gathers data from sensor nodes and send it to the sink node.

\section{B. Proposed System}

The proposed system contains cluster head, representative sensor nodes, isolated sensor nodes, member sensor nodes and these nodes send data to data center or cooperate node. 


\section{International Journal of Science and Research (IJSR) \\ ISSN (Online): 2319-7064 \\ Index Copernicus Value (2015): 78.96 | Impact Factor (2015): 6.391}

Then it calculates data density correlation degree and sends aggregated data to movable sink node or mobile sink.

Algorithm 1: Proposed Algorithm
1. Sense the environment
2. Selection of cluster head and it divide into clusters
3. Data gathering by cluster head
4. Data transmission by cluster head to data center/cooperate
node
5. Find data density correlation degree(DDCD)
6. Check energy consumption of every node
7. If lifetime of every node is over then data transfer by data
8. $\quad$ If lifetime of every node is not over then the node becomes
isolated node and repeat step 3
9. Check performance parameters and calculate result

Algorithm 1 gives steps for proposed algorithm. After sensing the environment, the data gathered by cluster head is transmitted to data center/cooperate node and then find the data density correlation degree. If lifetime of every node is over then data transfer by data center/cooperate node to mobile sink node and then check the performance parameters for calculating result. If lifetime of every node is not over then the node becomes isolated node and repeat the procedure to find DDCD.

\section{Mathematical Model}

A mathematical model of the proposed system is given below.

1. Total system $\mathrm{S}$ is defined as $\mathrm{DC}$

$\mathrm{S}=\{$

2. Identify input as

$\mathrm{I}=\{\varepsilon ; \operatorname{minPts} ; \mathrm{a} 1 ; \mathrm{a} 2 ; \mathrm{a} 3 ; N(i)\}$

$\varepsilon=$ Data Threshold

minPts $=$ Amount Threshold

a1; a2; a3 = Weights

$N(i)=$ Neighboring Sensor nodes set of Sensor Node $i$

3. Identify output as

$\mathrm{O}=\{\mathrm{R}\}$

$\mathrm{R}=$ Representative Sensor Nodes

4. Identify the sensor nodes $v$ has $n$ neighboring sensor nodes

$\mathrm{V}=v 1, v 2, \ldots, v_{n}$

5. Identify isolated sensor nodes, clusters as $C$ and sink nodes.

6. Identify the data object of $v$ is D. Its neighboring sensor nodes' data objects are respectively

$\mathrm{D}=\mathrm{D}_{1}, \mathrm{D}_{2}, \ldots, \mathrm{D}_{\mathrm{n}}$.

7. Using

$\operatorname{Sim}(v)=\left\{\begin{array}{c}0, \\ N<\min P t s \\ a_{1}\left(1-\frac{1}{\exp (N-\min P t s)}\right)+a_{2}\left(1-\frac{d_{\Delta}}{\varepsilon}\right)+a_{3}\left(1-\frac{d}{\varepsilon}\right), \\ N \geq \min P t s\end{array}\right.$

Identify the data density correlation degree of sensor node $v$ to the sensor nodes whose data objects are in $\varepsilon$ neighborhood of D.

8. Calculate delta for distance purpose (applied distance formula for neighboring count)

9. Identify neighbor count and check core sensor node.

10. NodeSetinner $(i)$ includes the IDs of the sensor nodes whose data are in the $\varepsilon$ - neighborhood of the data of sensor node $\mathrm{i}$.
11. NodeSetouter $(i)$ includes the IDs of the sensor nodes whose data are not in the $\varepsilon$ - neighborhood of the data of sensor node $i$.

12. Identify $\operatorname{Sim}(v)$ as Data Density Correlation Degree.

13. Using Data Density Correlation Degree, finds out the output as packet delivery ratio, dropping ratio, delay, throughput, normalized overhead, jitter and average energy consumption with respect to node and sense time.

\section{Experimental Setup}

The result of research and development is nothing but an elegant product name Network Simulator (NS). To build ns system needs a computer and a $\mathrm{C}++$ compiler. NS can install on several kinds of UNIX (FreeBSD, Linux, SunOS, and Solaris). NS comes with several packages inbuilt supporting Tcl/Tk files. The NS-2 simulation parameters are: Network are- 500x500, Topology-Flat grid, Routing protocolMDDCDC, No. of nodes-101, Initial energy-100 Joules, No. of sink nodes-3 and simulation sense time-35 sec, end time$190 \mathrm{sec}$.

\section{Results and Discussion}

In the proposed method, the aggregated data of data center/cooperate node is send to movable sink node/mobile node. The performance of proposed system is calculated by using packet delivery ratio, dropping ratio, delay, RSN and average energy consumption with respect to node and sense time.

In fig.2, the compared graph of packet delivery ratio with respect to node and sense time is given. The packet delivery ratio for proposed MDDCDC is higher than DDCDC method in both graphs. Fig. 2(a) shows node v/s PDR graph and fig. 2(b) shows sense time v/s PDR graph.

In fig.3, the compared graph of packet dropping ratio with respect to node and sense time is given. The dropping ratio for proposed MDDCDC is less than DDCDC method in both graphs. Fig. 3(a) shows node v/s dropping ratio graph and fig. 3(b) shows sense time $\mathrm{v} / \mathrm{s}$ dropping ratio graph.

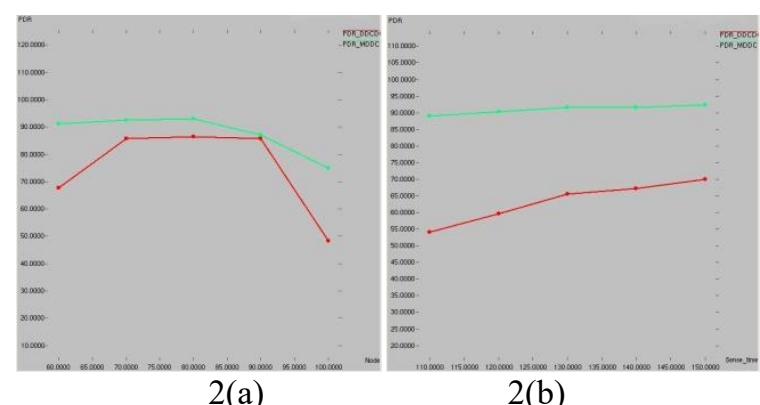

Figure 2: Packet delivery ratio(PDR). 2(a) Node v/s PDR, 2(b) Sense time v/s PDR graph 


\section{International Journal of Science and Research (IJSR) \\ ISSN (Online): 2319-7064}

Index Copernicus Value (2015): 78.96 | Impact Factor (2015): 6.391

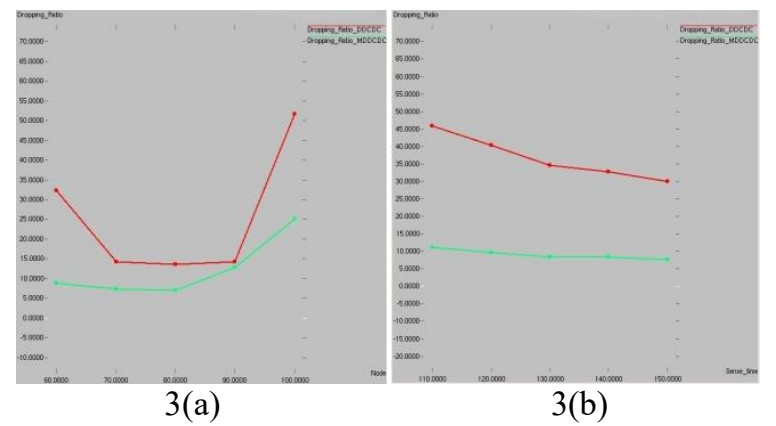

Figure 3: Dropping ratio. 3(a) Node v/s dropping ratio, 3(b) Sense time v/s dropping ratio graph

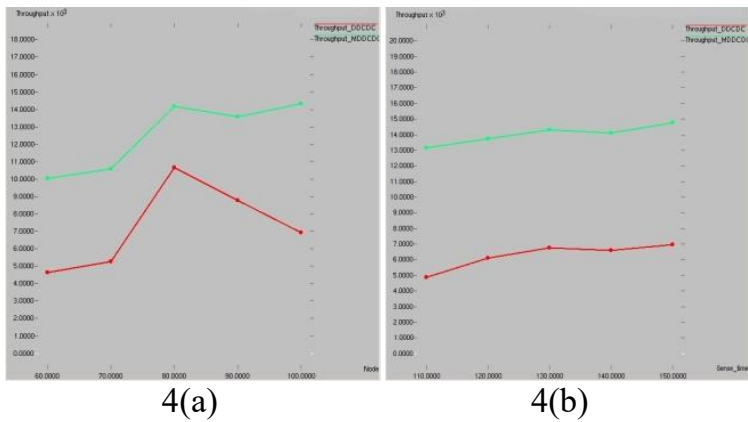

Figure 4: Throughput. 4(a) Node v/s throughput, 4(b) sense time $\mathrm{v} / \mathrm{s}$ throughput graph

In fig.4, the compared graph of throughput with respect to node and sense time is given. The throughput for proposed MDDCDC is higher than DDCDC method in both graphs. Fig. 4(a) shows node v/s throughput graph and fig. 4(b) shows sense time $\mathrm{v} / \mathrm{s}$ throughput graph.

In fig.5, the compared graph of representative sensor node (RSN) with respect to node is given. The RSN for proposed MDDCDC is much larger than DDCDC method. In MDDCD clustering method, the aggregated data is transferred from data center node to movable sink node i.e. mobile sink and this process, the representative sensor nodes is much larger. In existing DDCD clustering method, aggregated data is stored at data center node and also data density correlation degree is calculated.

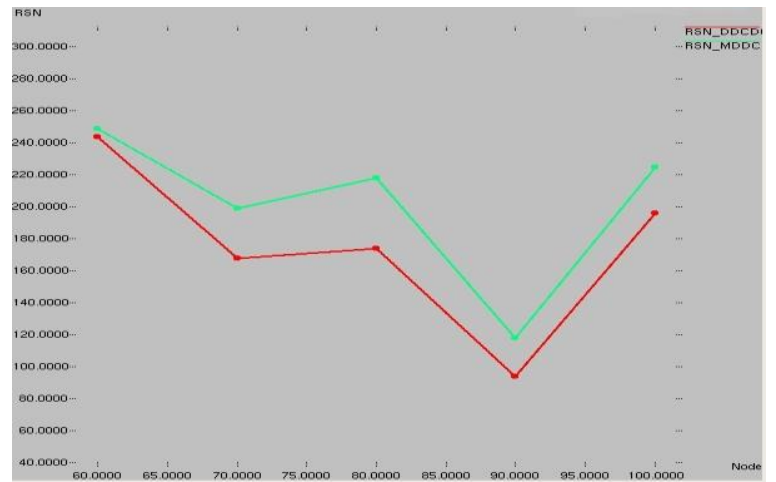

Figure 5: Node v/s representative sensor node(RSN) graph

In fig.6, the compared graph of average energy consumption with respect to sense time is given. The average energy consumption for proposed MDDCDC method is less than DDCDC method. The average energy consumption is measured in Joules and the initial energy is 100 Joules.

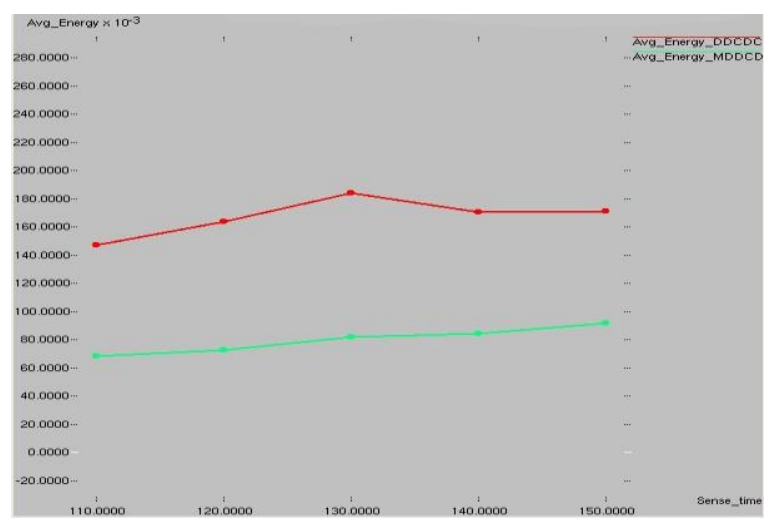

Figure 6: Sense time v/z average energy consumption graph

The sense time is measured in seconds and average energy consumed is measured in joules. The compared graph of sense time and average energy consumption is shown in fig. 6.

This system is related to some of the WSN applications such as event tracking may require sensor nodes to periodically perform observation and transmission of the sensed event features.

\section{Conclusion and Future Scope}

The proposed system reduces energy consumption with the use of cooperative node without affecting the other performance parameters. It achieves greater throughput, packet delivery ratio, packet loss ratio, delay, normalized overhead and representative sensor nodes with balanced energy consumption than the normal scenario i.e. without cooperative node. The performance optimization can be achieved in WSN using simulations. The proposed system uses a cooperate node which cooperates among the sensor nodes in a particular area of WSN and which improves transmission policies as well as energy efficiency.

In future work, DDCD clustering method can develop a method which could confirm the parameters adapted to the real sampled data.

\section{Acknowledgment}

The authors would like to thank the publishers, researchers for making their resources available and teachers for their guidance. We also thank the college authority for providing the required infrastructure and support. Finally, we would like to extend a heartfelt gratitude to friends and family members.

\section{References}

[1] Fei Yuan, Yiju Zhan, and Yonghua Wang, "Data Density Correlation Degree Clustering Method for Data Aggregation in WSN," IEEE Sensors Journal, Vol. 14, No. 4, April 2014.

[2] Ms. S.Sahana, Dr. R.Amutha, "Data Aggregation in Wireless Sensor Networks," IEEE, ICICES 2014.

[3] A.K.M. Azad, Student Member, IEEE, and Joarder Kamruzzaman, Member, IEEE, "Energy-Balanced Transmission Policies for Wireless Sensor Networks," 


\section{International Journal of Science and Research (IJSR) \\ ISSN (Online): 2319-7064}

Index Copernicus Value (2015): 78.96 | Impact Factor (2015): 6.391

IEEE Transactions on Mobile Computing, Vol. 10, No. 7, JULY 2011.

[4] Pu Wang and Cheng Li, Jun Zheng, "Distributed Data Aggregation Using Clustered Slepian-Wolf Coding in Wireless Sensor Networks," IEEE Communications Society, 2007.

[5] Yajie Ma, Yike Guo, Xiangchuan Tian, and Moustafa Ghanem, "Distributed Clustering-Based Aggregation Algorithm for Spatial Correlated Sensor Networks," IEEE sensors journal, vol. 11, no. 3, march 2011.

[6] Weiwei Fang, Feng Liu, Fangnan Yang, Lei Shu, and Shojiro Nishio, "Energy-Efficient Cooperative Communication for Data Transmission in Wireless Sensor Networks," IEEE, 2010.

[7] Dali Wei, Member, IEEE, Yichao Jin, Serdar Vural, Member, IEEE, Klaus Moessner, Member, IEEE, and Rahim Tafazolli, Member, IEEE, "An Energy-Efficient Clustering Solution forWireless Sensor Networks," IEEE Transactions on Wireless Communications, Vol. 10, No. 11, November 2011.

[8] Ramesh Rajagopalan, Pramod K. Varshney, "DataAggregation Techniques In Sensor Networks: A Survey," IEEE Communications Surveys \& Tutorials, Volume 8, No. 4, 4th Quarter 2006.

[9] Sk Md Mizanur Rahman, Mohammad Anwar Hossain, Maqsood Mahmud, Muhammad Imran Chaudry, Ahmad Almogren, Mohammed Alnuem, Atif Alamri, "A lightweight Secure Data Aggregation Technique for Wireless Sensor Network," 2014 IEEE International Symposium on Multimedia, 2014.

[10]Chengliang Wang, Yu Zhang, Wen-Zhan Song, "A New Data Aggregation Technique in Multi-sink Wireless Sensor Networks," SMARTCOMP 2014 / SES, 2014.

[11] G.Lydia Greeta Anandhi, K.Rajeswari, Dr. M. A. Bhagyaveni, "Cooperative Node Selection For Virtual Mimo In Wireless Sensor Networks," IJTES, Vol 3.No.1 Jan- March 2012.

[12]R.S.Janani, Mr.R.Shankar, Ms.M.Savitha, "Energy Balanced Wsn With Enhanced-Ddcd Clustering Method," IRJET, Volume: 02 Issue: 07 | Oct-2015.

[13] Y. Chalapathi Rao, Ch. Santhi Rani, "DDCD Algorithm based Energy Efficient Clustering for WSNs," Indian Journal of Science and Technology, Vol 9(29), August 2016.

[14] Kiran Maraiya, Kamal Kant, Nitin Gupta, "Efficient Cluster Head Selection Scheme for Data Aggregation in Wireless Sensor Network," International Journal of Computer Applications, Volume 23- No.9, June 2011.

[15]Haibo Zhang, Hong Shen, "Balancing Energy Consumption to Maximize Network Lifetime in DataGathering Sensor Networks," IEEE Transactions On Parallel And Distributed Systems, Vol. 20, No. 10, October 2009. 